\title{
VIABILIDADE ECONÔMICA DA CULTURA DO MELÃO (Cucumis melo L.) NA REGIÃO DE ILHA SOLTEIRA - SP ${ }^{1}$.
}

\author{
H.B. PAULINO²; M.A.A. TARSITANO; F.B.T. HERNANDEZ; S. BUZETTI ${ }^{3}$ \\ Faculdade de Engenharia de tha Solleira - FEIS/UNESP, C.P. 31, CEP: 15378-000 - Itha Solteira,SP
}

\begin{abstract}
RESUMO: Com o objetivo de se verificar a viabilidade económica da cultura do melăo, na região de Iha Solteira, ano agricola 1991/92, proceden-se a uma análise económica para estudar o comportamento de dois cultivares de melāo Eldorado 300 e Valenciano Amarelo CAC, variando a adubação de cobertura, ou seja, utilizando-se 3 doses de $\mathrm{N}$ e de $\mathrm{K}_{2} \mathrm{O}: 2,5 ; 5,0$ e 10,0 g/planta. $\mathrm{O}$ experimento foi irrigado atraves de um sistema de gotejamento, sendo que o investimento inicial para aquisiçăo do material de irrigaçāo para uma área de 1 ha, foi da ordem de USS 11.500,00. Apesar do alto valor do investimento inicial, a cultura se mostrou altamente rentável. Com os resultados obtidos, verificou-se que todos os tratamentos apresentaram rentabilidade positiva, sendo que a maior receita líquida encontrada foi de US\$ $\mathbf{4 . 0 4 8 , 0 0}$ do resultado económico, gerado pela diferença entre a receita bruta e o custo total, recebe influência direta dos níveis de produtividade e do preço alcançado pelo produto.

Descritores: custo de produção, sistema de produção, melăo
\end{abstract}

\section{ECONOMICAL, VIABILITY OP THE MELON CROP (Cucumis melo L.) IN THE ILHA SOLTEIRA REGION}

\begin{abstract}
To verify the economical viability of the melon crop in tha Solteira, this experiment was carried out during 1991/1992 performing an economical analysis of the behavior of two melon varieties: Eldorado 300 and Valenciano Amarelo CAC, varying the fertilizer dressing: $2.5 ; 5.0$ and $10.0 \mathrm{~g} /$ plant of $\mathrm{N}$ and $\mathrm{K}_{2} \mathrm{O}$. The experimental area was irrigated with a drop irrigation system, of an initial investment of US\$ $11,500.00$ per hectare. In spite of the high initial investment, the crop showed to be highly profitable. The obtained results show that the all treatments present positive productivity, with a high net income of US $\$ 4,048.00$ per hectare. The economic result cansed by the difference between the gross income and the total cost, is directly influenced by the productivity levels and the prices of the product obtained in the market.
\end{abstract}

Key Words: production cost, technology, muskmelon

\section{INTRODUÇÃO}

São vários os trabalhos enfocando a adubação e irrigação na produção e qualidade dos frutos do meloeiro, entre eles, citam-se COSTA (1987) e FERREIRA et al. (1982). No entanto, muitos desses trabalhos não foram interpretados em termos de recomendação econômica em nível de produtor.

É praticamente desnecessário ressaltar a importância da análise econômica em experimentação, pois não se pode sugerir a difusão de técnicas baseadas apenas nos resultados físicos. Há de se considerar os aspectos econômicos para a recomendação de práticas agrícolas mais adequadas.
A importância de tal estudo vem se acentuando principalmente nos últimos anos, quando os preços dos insumos tendem a crescer relativamente mais que os preços dos produtos. Daí a necessidade de os agricultores utilizarem tais insumos eficientemente para minimizarem os custos variáveis por unidade produzida.

A participação da irrigação na composição dos custos de produção é um dado importante. Além disso a análise dos índices de rentabilidade da cultura pode auxiliar no processo de escolha do sistema de produção. Deste modo, CALEGAR e COELHO (1987) procederam a uma análise econômica de dois métodos de irrigação: por sulco e gotejamento, em diversas condições de

\footnotetext{
1 Trabalho desenvolvido com o apoio financeiro da FAPESP e CNPq.

${ }^{2}$ Bolsista da FAPESP.

${ }^{3}$ Bolsista do CNPq.
}

Sci. agric., Piracicaba, 51(2):519-523, set./dez., 1994 
manejo, na cultura do melão, cultivar Valenciano Amarelo. Economicamente, os melhores resultados foram obtidos com irrigação por gotejo, com cerca de $55 \%$ de aumento na renda líquida, quando comparados aos resultados do método de irrigação por sulco.

Segundo EMBRAPA (1989), a área plantada nas regiões sudeste e nordeste no ano de 1986, foi de 387 e 3.370 ha, respectivamente, a produtividade média neste mesmo ano foi de 12,2 t/ha para a região sudeste, contra 6,4 t/ha para a região nordeste, o que faz com que o grande volume de produção se concentre na região Nordeste.

Entretanto, o grande consumo de frutos do meloeiro é feito na região sudeste, obrigando a exportação para os centros consumidores desta região, representando com isso um enorme aumento de preço do produto final. Segundo dados do CEAGESP (1986-1991) 45\% do volume comercializado no ano de 1991 foi proveniente do Rio Grande do Norte, e apenas 6\% do Estado de São Paulo.

A Figura 1 mostra a evolução da quantidade média de caixas de melão comercializadas por mês e preços médios praticados no CEAGESP/SP, no período de 1986 a 1991. Verifica-se que as maiores quantidades do produto são comercializadas no segundo semestre do ano, no qual também se verifica menores níveis de preços recebidos pelos produtores. De janeiro a junho nota-se que os preços sobem acentuadamente.

Segundo NAGAI (1987) o volume comercializado em nível nacional ultrapassa $60.000 \mathrm{t}$, dos quais mais de $60 \%$ são consumidos em São Paulo e $20 \%$ exportados. Assim, o melão passou de artigo importado para artigo de exportação. No entanto, fazem-se necessários trabalhos de pesquisa com a cultura, uma vez que estudos na região inexistem.

Neste contexto, a preocupação fundamental da presente pesquisa é estimar, para cada tratamento, o custo de produção e renda líquida da cultura do melão irrigado por gotejamento, para 2 cultivares: Eldorado $300 \mathrm{e}$ Valenciano Amarelo CAC.

\section{MATERIAL E METODOS}

Análise Econômica: $\mathrm{Na}$ estrutura de custo, existe a diferenciação clássica entre as estimativas de custo total e custo operacional. $\mathrm{Na}$ estrutura de custo total, todos os ítens de custo são incluídos e divididos em custos variáveis: sementes, fertilizantes, salários, defensivos, etc. e custos fixos: depreciação de máquinas, benfeitorias, despesas gerais e remuneração dos fatores de produção. Estes se referem aos juros sobre o capital fixo, terra e remuneração do empresário (ver por exemplo, NORONHA, 1990). A metodologia operacional utilizada pelo Instituto de Economia Agrícola da Secretaria da Agricultura de São Paulo, procura explicitar o total de despesas efetuadas pelo produtor no transcorrer do ciclo da cultura. A sua estrutura compõe-se dos custos variáveis e de uma parte dos custos fixos, representados pela depreciação de máquinas, implementos e benfeitorias. Considerações adicionais sobre custo operacional podem ser vistos em MATSUNAGA et al. (1976).

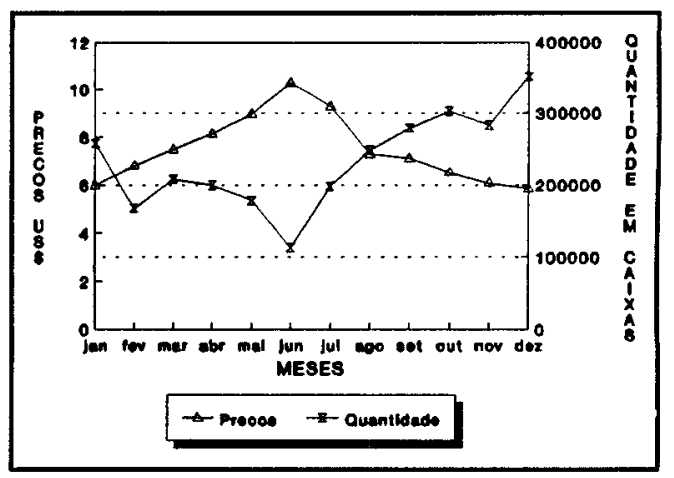

Figura 1 - Quantidade comercializada (caixas de $13 \mathrm{~kg}$ ) e preço médio mensal/caixa do melão praticados no CEAGESP (1986/1991).

A metodologia empregada para o cálculo do custo de produção do presente trabalho é baseada no custo operacional. Mais especificamente foram consideradas variáveis representadas por dispêndio com: salários, fertilizantes, defensivos, combustiveis, reparos e manutenção e juros de custeio. Adiciona-se a essas despesas uma parcela dos custos fixos representada pela depreciação de bens duráveis empregados no processo de produção.

\section{Levantamento dos Dados}

Visando atender o objetivo proposto de estudar a viabilidade econômica da cultura do meloeiro na região de Ilha Solteira, instalou-se um experimento na Fazenda de Ensino e Pesquisa da FEIS/UNESP, em um solo classificado como 
Podzólico Vermelho Escuro, eutrofico de textura arenosa, no ano agrícola de 1991/92.

Os cultivares utilizadas no experimento foram a Eldorado 300 e o Valenciano Amarelo CAC, sendo semeadas em espaçamento de 2,0 metros entre linhas e 0,75 metros entre plantas.

0 experimento foi irrigado por um sistema de irrigação por gotejamento, segundo as recomendações vigentes $e$ tendo como base a evaporação do Tanque Classe $A$, instalado no local do experimento.

O delineamento utilizado foi o de blocos ao acaso em um esquema fatorial $3 \times 3$ e com quatro repetições. Os tratamentos foram os seguintes: $\mathbf{N}_{1} \mathrm{~K}_{1}, \mathrm{~N}_{1} \mathrm{~K}_{2}, \mathrm{~N}_{1} \mathrm{~K}_{3}, \mathrm{~N}_{2} \mathrm{~K}_{1}, \mathbf{N}_{2} \mathrm{~K}_{2}, \mathbf{N}_{2} \mathrm{~K}_{3}$, $\mathrm{N}_{3} \mathrm{~K}_{1}, \quad \mathrm{~N}_{3} \mathrm{~K}_{2}, \quad \mathrm{~N}_{3} \mathrm{~K}_{3}$, onde os níveis 1,2 e 3 expressam as doses de 2,5; 5,0 e 10,0 gramas/ planta, em cada cobertura, de $\mathrm{N}$ e $\mathrm{K}_{2} \mathrm{O}$, respectivamente. As adubações de cobertura foram feitas aos 15,30 e 45 dias após a emergência das plantas e todos os tratamentos receberam adubação de plantio que constou de $300 \mathrm{~g} /$ cova da fórmula 04-30-10. As parcelas foram constituídas de 3 linhas e 5 plantas por linha e as avaliações foram realizadas na linha central, extraindo-se uma planta de cada extremidade.

A condução das plantas foi realizada de forma natural, sem podas e desbaste de frutos, e o controle fitossanitário de acordo com as recomendações para um bom desenvolvimento das plantas, de modo a proporcionar produção de frutos saudáveis e de boa qualidade.

Para se proceder a avaliação econômica da pesquisa, foram acompanhadas, para estimativas de custo, todas as operações de implantação, condução e colheita da cultura.

Definição das variáveis dos custos de produção: As bases de cálculo para estas estimativas são as matrizes de coeficientes técnicos coletadas junto ao experimento e a produtores da região, para que se obtivesse dados os mais reais possíveis. As operações envolvidas compreendem todo o ciclo da cultura, ou seja, desde a instalação do equipamento de irrigação por gotejamento, até a condução da cultura e colheita.

Inicialmente procedeu-sea um levantamento das necessidades de mão-de-obra nas diversas fases do ciclo produtivo relacionando-se, para cada operação a ser realizada, o número de homens dias (HD) para executá-lo. Em seguida multiplica-se estes coeficientes técnicos de necessidade de mãode-obra pelo salário médio da região.
Para determinação do custo da hora de irrigação por gotejamento adotou-se como componente do custo fixo a depreciação, e como custos variáveis a energia de consumo e os reparos e manutenção com o equipamento. $O$ gasto com energia foi obtido pelo consumo do motor de $5 \mathrm{cv}$ utilizado na pesquisa. $O$ valor do $\mathrm{Kw} . \mathrm{h}(\mathrm{Cr} \$ 23,75)$ se refere ao mês de outubro de 1991, segundo dados apresentados pela CESP de Ilha Solteira. Deste modo o custo horário da irrigação foi composto de despesas com energia (Cr\$89,00/hora), mais despesas com reparos e manutenção ( $\mathrm{Cr} \$ 48,00 /$ hora) e depreciação do equipamento (Cr\$388,00/ha).

Nos custos com máquinas foram consideradas as despesas com combustível, lubrificantes, reparos e manutenção, alojamento e depreciação. A depreciação refere-se à perda do valor de um bem de capital. O método usado foi o linear, muito embora apresente certos inconvenientes como os mostrados por HOFFMANN (1976). Portanto, a depreciação foi calculada pela diferença entre o valor inicial e o residual final do bem de capital, dividido pela vida útil dos mesmos. Os gastos com insumos referem-se às despesas com adubos, defensivos, etc. e foram conseguidos mediante o produto entre a quantidade dos fatores de produção usados $e$ os seus respectivos preços unitários.

Os custos foram determinados através dos preços pagos na região em junho de 1993, e foram convertidos em dólar norte-americano, para análises em moeda constante.

\section{RESULTADOS E DISCUSSÃO}

Na TABELA 1 consta a relação de material necessário para irrigar 1 ha de melão por gotejamento, além do investimento total de US\$ $11.500,00$, orçamento fornecido pela firma comercializadora do equipamento.

Para exemplificar, a TABELA 2 apresenta a estimativa de custo de produção e receita da cultura do meloeiro, cv Eldorado 300, detalhada em seus diversos componentes. Deve-se salientar que apesar do alto investimento inicial, com o equipamento de irrigação por gotejamento, as despesas com energia são menores quando comparadas com o sistema de irrigação convencional.

As despesas de custeio (operações + insumos) atingem US $\$ 2.088,54$ e de todos os ítens que compõem o custo, destaca-se em maior proporção os gastos com operações manuais, representando cerca de $60 \%$, mostrando ser a cultura do meloeiro muito exigente em termos de mão-de-obra. 
TABELA 1 - Relação de material necessário para irrigar 1 ha de melão por gotejamento - Outubro de 1991.

\begin{tabular}{|c|c|c|}
\hline \multirow{2}{*}{$\begin{array}{l}\text { Material } \\
\text { Gotejador Katif 2,3" }\end{array}$} & \multicolumn{2}{|c|}{ Unidade Quantidade } \\
\hline & Ps & 6.700 \\
\hline $\begin{array}{l}\text { Tubo PE 12,5" } \\
\text { Uniäo interna 12,5" }\end{array}$ & $\begin{array}{l}\mathbf{m} \\
\mathrm{pc}\end{array}$ & $\begin{array}{r}5.500 \\
30\end{array}$ \\
\hline Adaptador para mangueira & pq & 80 \\
\hline Final de linha $12,5^{\prime \prime}$ & pq & 80 \\
\hline Tubo de PVC $75 \mathrm{~mm}$ PN 80 PB & $\mathbf{m}$ & 300 \\
\hline Tubo de PVC $75 \mathrm{~mm}$ PN $80 \mathrm{JE}$ & $\mathbf{m}$ & 120 \\
\hline Adaptador $60 \mathrm{~mm}$ PVC & pq & 9 \\
\hline Bucha de redução $75 \times 60 \mathrm{~mm}$ & pq & 3 \\
\hline Bucha de redução $65 \times 25 \mathrm{~mm}$ & pq & 1 \\
\hline Curva de $45 \times 75 \mathrm{~mm}$ & p̧ & 2 \\
\hline Niple PVC 3/4" & pq & 40 \\
\hline Luva de PVC R 1/2" & $\mathrm{pc}$ & 75 \\
\hline Curva de $90 \times 75 \mathrm{~mm}$ & pç & 3 \\
\hline Curva de $90 \times 60 \mathrm{~mm}$ & $\mathrm{pc}$ & 4 \\
\hline Flange de $60 \mathrm{~mm}$ & $\mathrm{pc}$ & 4 \\
\hline Capa PVC $75 \mathrm{~mm}$ & $\mathrm{pq}$ & 1 \\
\hline Capa PVC $65 \mathrm{~mm}$ & $\mathrm{pc}$ & 2 \\
\hline Bucha redução 3/2" & p̧̧ & 2 \\
\hline Bucha redução $2 \times 1 / 2$ " & pc & 1 \\
\hline Curva MF de 2" & pç & 1 \\
\hline Niple de 2" & $\mathrm{pc}$ & 14 \\
\hline Niple de 1 1/2" & pç & 1 \\
\hline União de $2 "$ & p̧ & 3 \\
\hline Tê de $2 "$ & $\mathrm{pc}$ & 3 \\
\hline Luva de redução $3 \times 2 "$ & $\mathrm{pc}$ & 1 \\
\hline Registro de gaveta 2" & p̧ & 4 \\
\hline Válvula D-228 & pq & \\
\hline Ventosa de 1" & p̧̧ & 1 \\
\hline Antivácuo de 1/2" & pç & 2 \\
\hline Filtro de areia $600 \mathrm{~mm}$ & pq & 2 \\
\hline Filtro de disco de $2 "$ & $\mathrm{pc}$ & \\
\hline Tanque de fertilização 401 & pc & 1 \\
\hline Manômetro 0-10 & pç & 2 \\
\hline Areia Lavada & pc & 8 \\
\hline Bomba e Motor & pq & 1 \\
\hline Chave de Partida & pç & 1 \\
\hline
\end{tabular}

Custo Total $=$ Cr $\$ 6.900 .000,00$ (US\$ $11.500,00)$

O custo total de produção do meloeiro, segundo metodologia descrita foi de US\$2,392.29. Deste total, $60 \%$ aproximadamente corresponde a despesas com operações (mecanizada mais manuais) $27 \%$ gastos com insumos; juros bancários mais depreciação representou cerca de $13 \%$ (onde a participação maior se refere a depreciação do equipamento de irrigação). $O$ resultado econômico, gerado pela diferença entre a receita bruta e o custo total, recebe influência direta dos níveis de produtividade e do preço alcançado pelo produto em outubro/91.
TABELA 2 - Estimativa da renda líquida em US\$ ha e da taxa de retorno por tratamento na produção de melão.

Tratamento Produção Receita Custo Receita Taxa de Bruta Total Líquida Retorno

Valenciano Amarelo CAC

$\begin{array}{lllllll}1 & \mathrm{~N}_{1} \mathrm{~K}_{1} & 24.557 & 6.139,25 & 2.340,21 & 3.799,04 & 2,62\end{array}$

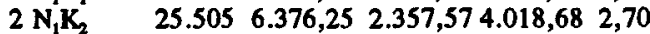

$\begin{array}{lllllll}3 \mathrm{~N}_{1} \mathrm{~K}_{\mathrm{s}} & 18.503 & 4.625,75 & 2.401,762.223,99 & 1,92\end{array}$

$\begin{array}{llllllll}4 & \mathrm{~N}_{2} \mathrm{~K}_{1} & & 17.521 & 4.380,25 & 2.340,21 & 2.040,04 & 1,87\end{array}$

$\begin{array}{llllll}5 \mathrm{~N}_{2} \mathrm{~K}_{2} & 17.659 & 4.414,75 & 2.357,572.057,18 & 1,87\end{array}$

$6 \mathrm{~N}_{2} \mathrm{~K}_{3} \quad 22.946 \quad 5.736,50 \quad 2.392,293.344,21 \quad 2,39$

$\begin{array}{lllllll}7 \mathrm{~N}_{3} \mathrm{~K}_{1} & 19.960 & 4.990,00 & 2.340,21 & 2.649,79 & 2,13\end{array}$

$\begin{array}{llllll}8 & \mathrm{~N}_{3} \mathrm{~K}_{2} & 19.283 & 4.820,75 & 2.357,572.463,18 & 2,04\end{array}$

$\begin{array}{llllll}9 \mathrm{~N}_{3} \mathrm{~K}_{3} & 18.358 & 4.589,50 & 2.392,29 & 2.197,21 & 1,91\end{array}$

Eldorado 300

$\begin{array}{llllll}1 & N_{1} K_{1} & 20.367 & 5.091,75 & 2.340,212.751,54 & 2,17\end{array}$

$\begin{array}{lllllll}2 \mathrm{~N}_{1} \mathrm{~K}_{2} & 15.567 & 3.891,75 & 2.357,57 & 1.534,18 & 1,65\end{array}$

$3 \mathrm{~N}_{1} \mathrm{~K}_{3} \quad 23.6505 .912,502.401,763.510,742,46$

$\begin{array}{lllllll}4 \mathrm{~N}_{2} \mathrm{~K}_{1} & 21.841 & 5.460,25 & 2.340,21 & 3.120,04 & 2,33\end{array}$

$\begin{array}{llllll}5 \mathrm{~N}_{2} \mathrm{~K}_{2} & 24.144 & 6.036,00 & 2.357,573.678,43 & 2,56\end{array}$

$\begin{array}{lllllll}6 \mathrm{~N}_{2} \mathrm{~K}_{3} & 16.031 & 4.007,75 & 2.392,29 & 1.615,46 & 1,67\end{array}$

$\begin{array}{lllllll}7 \mathrm{~N}_{3} \mathrm{~K}_{1} & 21.098 & 5.274,50 & 2.340,21 & 2.934,29 & 2,25\end{array}$

$\begin{array}{llllll}8 \mathrm{~N}_{3} \mathrm{~K}_{2} & 22.850 & 5.712,50 & 2.357,573.354,93 & 2,42\end{array}$

$\begin{array}{llllll}9 \mathrm{~N}_{3} \mathrm{~K}_{3} & 25.761 & 6.440,25 & 2.392,29 & 4.047,96 & 2,69\end{array}$

O retorno econômico calculado indica que a rentabilidade envolvida no processo de produção pode ser substancial. Em todos os tratamentos a rentabilidade foi positiva, assim, para uma produção de $25.761 \mathrm{Kg} / \mathrm{ha}$, comercializada a preço de mercado (Cr\$ $150,00 / \mathrm{Kg}$ ), o retorno líquido atinge US\$ 4.047,96/ha.

As estimativas de custo de produção do melão, receita, renda e produção para os 9 tratamentos dos cultivares Valenciano Amarelo CAC e Eldorado 300, se encontram resumidas na TABELA 3, as despesas com operações são iguais para todos os tratamentos, só variando os gastos com insumos (doses de $\mathrm{N}$ e de $\mathrm{K}_{2} \mathrm{O}$ ). $\mathrm{O}$ resultado obtido com o cultivo, mostra um rendimento superior do Eldorado 300 , em relação ao Valenciano Amarelo CAC, resultando em maior renda líquida por ha.

Analisando o retorno líquido obtido pe. los tratamentos (TABELA 3) verifica-se que o tratamento $2\left(\mathrm{~N}_{1} \mathrm{~K}_{2}\right)$ e o tratamento $9\left(\mathrm{~N}_{3} \mathrm{~K}_{3}\right)$ dos cultivares Valenciano Amarelo CAC e Eldorado 300, respectivamente alcançaram as maiores taxas de retorno. No geral, as taxas de retorno obtidas com a cultivar Eldorado 300 são maiores que as obtidas com o Valenciano Amarelo. Assim, dependendo do nível de produtividade alcançado a taxa de retorno varia de 1,65 a 2,70 . Isto quer dizer que do total de gasto no custo total o retorno é de 65 a $170 \%$. 
TABELA 3 - Produção (Kg/ha), estimativa de custo, receita e taxa de retorno por tratamento da cultura do meloeiro em US\$/ha, outubro de 1991.

\begin{tabular}{lll}
\hline $\begin{array}{l}\text { Trata- Produção custo } \\
\text { mento }\end{array}$ & total toceita & $\begin{array}{c}\text { receita taxa de } \\
\text { líquida retorno }\end{array}$
\end{tabular}

cv. Valenciano Amarelo - CAC

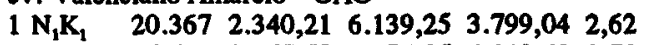

$\begin{array}{lllllll}2 & \mathrm{~N}_{1} \mathrm{~K}_{2} & 15.597 & 2.357,57 & 6.376,25 & 4.018,68 & 2,70\end{array}$

$\begin{array}{llllll}3 \mathrm{~N}_{1} \mathrm{~K}_{3} & 18.503 & 2.401,76 & 4.625,75 & 2.223,99 & 1,93\end{array}$

$\begin{array}{llllll}4 \mathrm{~N}_{2} \mathrm{~K}_{1} & 17.521 & 2.340,21 & 4.380,25 & 2.040,04 & 1,87\end{array}$

$\begin{array}{llllll}5 \mathrm{~N}_{2} \mathrm{~K}_{2} & 17.659 & 2.357,57 & 4.414,75 & 2.057,18 & 1,87\end{array}$

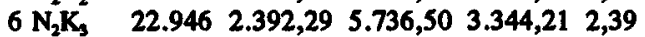

$\begin{array}{llllllll}7 & \mathrm{~N}_{3} \mathrm{~K}_{1} & 19.960 & 2.340,21 & 4.990,00 & 2.649,79 & 2,13\end{array}$

$\begin{array}{llllllll}8 \mathrm{~N}_{3} \mathrm{~K}_{2} & 19.283 & 2.357,57 & 4.820,75 & 2.463,18 & 2,04\end{array}$

$\begin{array}{llllll}9 \mathrm{~N}_{3} \mathrm{~K}_{3} & 18.358 & 2.392,29 & 4.589,50 & 2.197,21 & 1,92\end{array}$

cv. Eldorado 300

$\begin{array}{llllll}1 \mathrm{~N}_{1} \mathrm{~K}_{1} & 24.557 & 2.340,21 & 5.091,75 & 2.751,54 & 2,18\end{array}$

$\begin{array}{lllllll}2 \mathrm{~N}_{1} \mathrm{~K}_{2} & 25.505 & 2.357,57 & 3.891,75 & 1.534,18 & 1,65\end{array}$

$\begin{array}{llllllllll}3 \mathrm{~N}_{1} \mathrm{~K}_{3} & 23.650 & 2.401,76 & 5.460,25 & 3.510,74 & 2,46\end{array}$

$\begin{array}{lllllll}4 \mathrm{~N}_{2} \mathrm{~K}_{1} & 21.841 & 2.340,21 & 5.912,50 & 3.120,04 & 2,33\end{array}$

$\begin{array}{lllllll}5 \mathrm{~N}_{2} \mathrm{~K}_{2} & 24.144 & 2.357,57 & 6.036,00 & 3.678,43 & 2,56\end{array}$

$\begin{array}{lllllll}6 & \mathrm{~N}_{2} \mathrm{~K}_{3} & 16.031 & 2.392,29 & 4.007,75 & 1.615,46 & 1,67\end{array}$

$\begin{array}{lllllll}7 & \mathrm{~N}_{3} \mathrm{~K}_{1} & 21.098 & 2.340,21 & 5.274,50 & 2.934,29 & 2,25\end{array}$

$\begin{array}{lllllll}8 & \mathrm{~N}_{3} \mathrm{~K}_{2} & 22.850 & 2.357,57 & 5.712,50 & 3.354,93 & 2,42\end{array}$

$\begin{array}{llllll}9 \mathrm{~N}_{3} \mathrm{~K}_{3} & 25.761 & 2.392,29 & 6.440,25 & 4.047,96 & 2,69\end{array}$

\section{CONCLUSÕES}

No geral as taxas de retomo obtidas com o cultivar Eldorado $\mathbf{3 0 0}$ foram maiores do que as obtidas com a cultivar Valenciano Amarelo CAC, mas todos os tratamentos apresentaram Renda Líquida positiva.

As tabelas apresentadas permitem avaliar os efeitos nos custos de implantação e produção, em função de mudanças nos coeficientes técnicos utilizados. $O$ resultado econômico também pode ser alterado dependendo do nível de produção alcançado e do preço do melão. No presente estudo, observou-se que a maior taxa de retorno apresentada pelas dois cultivares foi de $170 \%$, sendo que para a cultivar Valenciano Amarelo CAC, o tratamento que apresentou este valor foi o tratamento $\mathrm{N}_{1} \mathrm{~K}_{2}$, o mesmo acontecendo com o tratamento $\mathrm{N}_{3} \mathrm{~K}_{3}$ da cultivar Eldorado 300 .

Apesar do alto investimento inicial com o equipamento de irrigação por gotejamento, o meloeiro se mostra altamente rentável, fato comprovado pelo sucesso de alguns agricultores na região.

Esta pesquisa ostenta algumas limitações, principalmente na aplicabilidade das conclusões obtidas. Ao planejar um experimento, deve-se verificar que existem variações de resposta que podem ocorrer de uma Epoca para outra e de um lugar para outro. A produtividade pode variar muito de um ano para o outro, dependendo das condições climáticas e o agricultor sempre trabalhar em função de expectativas de preços. Assim, sugere-se a implantação de novos experimentos para verificar se esses resultados são ou não confirmados.

\section{REFERENCIAS BIBLIOGRÁFICAS}

CALEGAR, G.M.; COELHO, M.B. Consideraçžes econômicas dos métodos de irrigaçũo por gotejamento e por sulco na cultura do melão. Petrolina: CPATSA/EMBRAPA, 1987. 11p.

CEAGESP. Melão: preço médio e quantidades mensais 1986-1991. Departamento de Economia. São Paulo.

COSTA, J.E.S. Análise físico-química do melâo produzido em condiçōes de casa de vegetação e irrigado pelos sistemas de jato-pulsante e gotejamento. Jaboticabal, UNESP-FCAV, 1987, 65p. (Trabalho de Graduação).

EMBRAPA. Área irrigada, produção e produtividade das principais hortalicas no Brasil - 1985/1986. CNHP: Brasilia, 1989. 62p. (Documento, 4)

FERRERA, F.A.; PEDROSA, J.F.; ALVAREnga, M.A.R. Melăo: cultivares e métodos culturais. Informe Agropecuário, Belo Horizonte, v.8, n.85, p.26-28, 1982.

HOFFMANN, R. Administraçāo da empresa agrícola. Editora Pioneira, São Paulo. 1976. 325p.

MATSUNAGA, M. Metodologia de custo de produção utilizada pelo Instituto de Economia Agrícola. Revista Agricultura em Săo Paulo, São Paulo, v.23, p.123139, 1976.

NAGAI, H. Instruçoes agricolas para o Estado de São Paulo: melāo. Campinas, Instituto Agronômico, 1987. p.145. (Boletim, 200).

NORONHA, J.F. Custos de produção e ańlilise econômica da atividade leiteira. SIMPÓsIO SOBRE PRODUÇÃO ANIMAL, 7, 1990, Campinas. Anais... Piracicaba: FEALQ, 1990. p.109-139.

Enviado para publicação em 09.08 .93

Aceito para publicaçắo em 24.09 .93 